\title{
Research on Pair Learning Method and Pattern Based on Pair Programming
}

\author{
Yue Zhang /per 1st Affiliation \\ Nanjing Normal University \\ Nanjing, Jiangsu, China \\ yueyuez697@163.com \\ Yang Yang / per 2nd Affiliation \\ Nanjing Normal University \\ Nanjing, Jiangsu, China \\ 15950560419@163.com
}

\author{
YeBo Yang / per 2nd Affiliation \\ Nanjing Normal University \\ Nanjing, Jiangsu, China \\ 15651607802@163.com \\ WanFeng Dou / per 3rd Affiliation \\ Nanjing Normal University \\ Nanjing, Jiangsu, China \\ douwanfeng@njnu.edu.cn
}

\begin{abstract}
Pair programming is an agile practice that supports two people working together locally or remotely to complete a programming task. Based on the cooperative learning theory, this paper puts forward the concept of pair learning and explains the method of pair learning from the aspects of pedagogy, psychology and game theory. We use the model that two individuals make up a pair and two pairs make up a large group. Based on the distributed pair system XPairtise, we carry out experiments and analyze the effect of different combination of characters, gender, ability and so on.
\end{abstract}

Keywords—pair learning; cooperative learning; pair learning method

\section{INTRODUCTION}

Pair programming refers to two programmers sitting in front of the computer to discuss the design schemes and algorithms, complete the code and a variety of tests, which is one of the practical methods of agile development [1]. At present, the method of pair programming is introduced into the practice of computer teaching, which is helpful to improve students' programming ability and comprehensive quality.

Cooperative learning is a learning model that began in the United States in the early 1970s. It is composed of 2-6 students to accomplish the goal of group learning, promoting the level of each member and improving the overall performance in a cooperative way. In the late 1980s and early 1990s, China has also developed research and experimentation of cooperative learning [4]. In this paper, we will combine pair programming method with cooperative learning and pedagogy concept. We propose a cooperative learning method that supports two individuals study in pairs, namely the method of pair learning. Also, theoretical methods and influencing factors that affect the pair learning are analyzed.

\section{PAIR LEARNING}

Pair programming greatly improves the efficiency and quality of programming, which is a good practice model. Cooperative learning also uses the concept of mutual assistance. However, cooperative learning commonly has a lot of people. The scale is large so the greatest problem may happen is whether all people are well involved cannot be ensured. Opportunity is reduced accordingly within a limited time. In addition, the number of members will lead to the complex structure of the interaction, necessarily increasing the requirement of cooperation skill [3].

Instead, pair programming is a cooperative learning model of two individuals. People play both strengths and complete the pair work through communication efficiently. One of the parties is called the driver, responsible for coding while the other side is called the navigator, responsible for checking the code. The navigator points out the wrong place and discuss with the driver to put forward improvement suggestion. Two sides regularly exchange roles in order to give full play to both advantages of them. At the same time with cooperative programming, knowledge exchange is also an important harvest. Through mutual discussion the two sides exchange knowledge so as to learn from each other's strengths. So, we consider a pair as a cooperation unit of the highest efficiency, we call it pair learning. Pair learning is based on the concept of pair programming and cooperative learning. The process of pairing is also the spread of knowledge, which can achieve the goal of mutual learning.

According to the actual impact of academic achievement, the American psychologist Ausubel divided the learning motivation into three categories: cognitive internal drive, ego-enhancement drive and affiliated drive [5]. At the same time, the American psychologist Yerkes and Dodson found that the working efficiency is the highest when the motivation is in an appropriate level [6];

Fund Project: Education Department of Jiangsu Province: Graduate student Innovation Project "Implementation and practice of pair learning based on pair programming" (JGLX15_133) 
If the motivation intensity is too low or too high, the enthusiasm will be rather low. Therefore, for leaders, when arranging for the tasks of the learning groups, they can consider the task as a purpose and properly adjust the different students to form learning groups. Leaders should arrange for hierarchical tasks for different groups. For the team that has the strong learning ability, they can appropriately increase the difficulty of the task. For others, they may appropriately reduce the difficulty of the task to boost the enthusiasm of the team.

In Game theory, cooperative and non-cooperative game, repeated game etc. are also instructive for pair learning. Pair programming mainly applies in software development and implementation of project. It is a cooperative game and it pays attention to the final distribution of income. Pair learning focuses more on mastering the knowledge that it pays attention to the choice of the strategy in the process. The game of pair programming and pair learning can also be seen as a repetitive game. In order to reduce some unnecessary friction, it can proceed from the rules and guide the two pairs to work consciously towards cooperation and winwin direction. Teachers can guide students in the time of pair learning, emphasize the construction of the relationship of the two and deal with the problem, etc. Enterprises can also carry out pair training for staffs.

\section{PAIR LEARNING METHODS AND PRACTICE}

\section{A. Pair Learning Methods.}

To make the pairing personnel with more sense of belonging, the opportunity of participating and communicating, we use the model of a group of two pairs, a large group of four people to simplify the structure of cooperation.

Strategy of Two-Individuals Combination. Efficient partner combinations are usually based on a combination of personality, gender, and competency. The combination of different personality and gender may produce different learning effect. According to the level of skill, the students are divided into high-level, intermediate-level and low-level three levels. Different abilities of students can get different gains in the process of pair learning.

Strategy of Two-Pairs Combination. Pair learning of two individuals may lead to lower heterogeneity within the group so that it is difficult to produce good thinking and ideas. So, learning from the theory of cooperative learning, we put two pairs into a group of four to increase the path of communication. To make up a group of four, we can use "Group Leader Method", "Social Relations Method" and "Random Team Method". Among them, "Group Leader Method" is commonly used. It is headed by a member who is more professional and he selects the team members according to the ability, high capacity with low ability.

Strategy of Relationship Construction. After the formation of the group, the relationship between the partners needs to be built so as to facilitate the latter part of the cooperation. This is an indispensable link in the success of pair cooperation. By relationship building, members feel understood and accepted.

The two must be familiar with each other at the beginning. Then they can simply communicate from their own preferences and other aspects. Then need to care about "We" rather than "I". At the same time, after the formation of the group, we need to let the team members recognize the differences between each other and respect these diversities. Group members can first solve some of the problems together to prepare later cooperation. Finally, we can use some small games to strengthen the mutual trust between the players and deepen the understanding.

Implementation Method. First of all we should create a suitable environment for pair learnig which makes us learn in a good atmosphere. Learning habits is also important that paticipants should learn to listen and express. Many ways can be used in pairing process. "Take Turns to Say", "Chains of Thinking", "Debate", "Peer Counselling Method", "Brainstorming" and so on have different effect. We generally do not limit the way we discuss in pairing experiments, but "Take Turns to Say" and "Peer Counselling Method" are more common and practical. The form of "Take Turns to Say" is that two or four members think independently first and then they explain in turns. The partner complements.

"Peer Counselling Method". The counselor demonstrated first, then the counselor tries. When error occurs, the counselor points out the error, let the counselor correct it on his own first, and then the counselor correct the error again, this process repeated [8].

\section{B. Pair Learning Process.}

The main process of pair learning practice is divided into five steps: personality questionnaire test, capacity evaluation, student grouping, in-class testing, data collection and analysis. The detailed process of each step is shown in Fig. 1.At the first and second stage of the experiment, we tested and classified the students' character and ability. Then in the experimental stage of grouping, according to personalities, genders, abilities, and the number of each group we had different combinations so that we could effectively carry out controlled trials. For example, according to the character of the personality test, we divided the students into three combinations: Introvert - Introvert, Introvert - Extrovert, Extrovert - Extrovert; according to gender, we divided them into three: Boy - Boy, Boy - Girl, Girl - Girl. Then during in-class testing phase, we released the same test and graded each group. Finally, in data collection and analysis phase, we analyzed from the test results obtained in various combinations and determined which combinations of pairing could make us learn more efficiently and provide reference. 


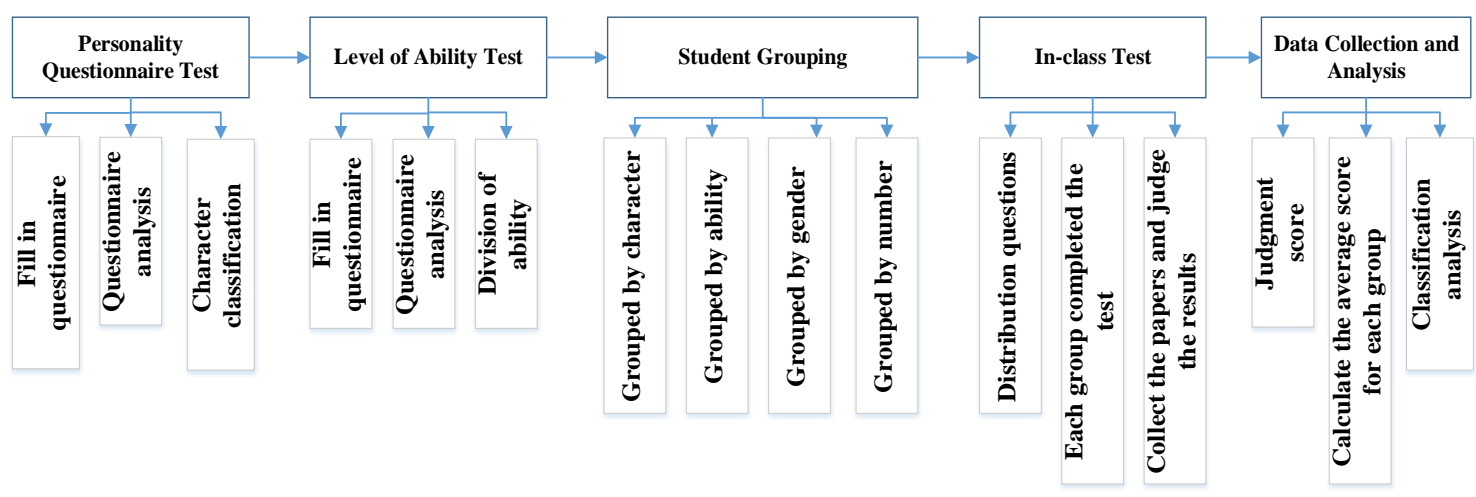

Fig.1. Experimental process

Pair Learning Experiment. In practice, it is often difficult to judge the various combinations. Therefore from the pairing process and results, we develop a set of performance assessment system. We evaluate the pairing process (such as whether the members have effective communication or a certain degree of progress, and so on) by recording real-time communication and giving the actual score. And we mainly evaluate the efficacy of the pair through the code accuracy.

We use the Xpairtise tool, which is now an open source project popular both at home and abroad, providing shared editing, project synchronization, program sharing, test execution, user management, embedded dialogue and shared whiteboard. [7] Through this platform pair programming can be done in different places.

We conducted more than three experiments in the Java programming community of Nanjing Normal University and Nanjing Normal University Affiliated School. Each student needs to complete the 8 programming questions with difficulty gradually enhanced. In this way can our experimental result be accurate so that we can analyze whether students get real progress. Table 1 was the data that obtained by testing.

Table1. $\quad$ EXPERIMENTAL DATA

\begin{tabular}{|c|c|c|}
\hline Category & Type & Number of people \\
\hline \multirow{3}{*}{ Division of personality tests } & Introvert & 223 \\
\cline { 2 - 3 } & Neutral & 387 \\
\cline { 2 - 3 } & Extrovert & 673 \\
\hline \multirow{2}{*}{ Gender division } & Boy & 409 \\
\cline { 2 - 3 } & Girl & 49 \\
\hline \multirow{2}{*}{ Test classification } & Senior & 508 \\
\cline { 2 - 3 } & Intermediate & 525 \\
\cline { 2 - 3 } & Primary & 154 \\
\hline \multirow{2}{*}{ Group size } & Single & 308 \\
\cline { 2 - 3 } & Pair & 620 \\
\cline { 2 - 3 } & Two pairs & 1082 \\
\hline
\end{tabular}

IV. ANALYSIS OF RESULTS

We get the average accuracy of different combinations. Fig. 2-5 reflects the results.

Random combination of different characters has been shown in Fig. 2. Extrovert - Neutral, Introvert - Neutral and Neutral Neutral, these three forms have the relatively high accuracy, while Introvert - Introvert form has the worst effect. During the experiment, from the communication record in the XPairtise plug-in we also found that communication effect between two introverted individuals is not good generally. Extrovert - Extrovert, this combination is also likely to lead to two students' excessive exchange instead of focus, so the effect is poor.

Combination of different character has been shown in Fig. 3. and accuracy of the combinations is almost the same that the highest and lowest differs by only $5 \%$. The highest average accuracy combination is Boy - Girl combination while combination of male - male has a lower efficiency and enthusiasm to study. The main reason is that boys and girls differ in the way of thinking so that they can complement each other in learning aspect. In addition, part of the students also express that cooperating with the opposite sex can increase their learning enthusiasm to some extent. 


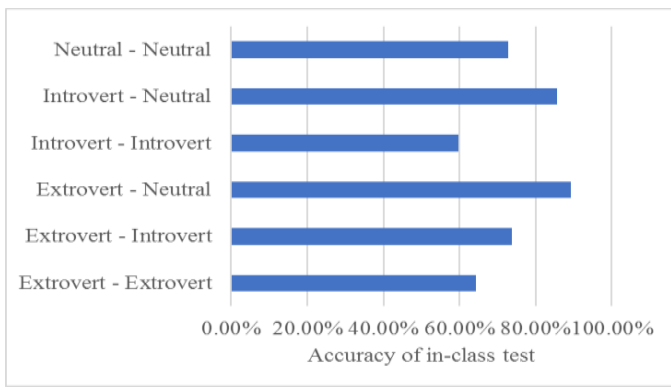

Fig. 2. Results of different personalities

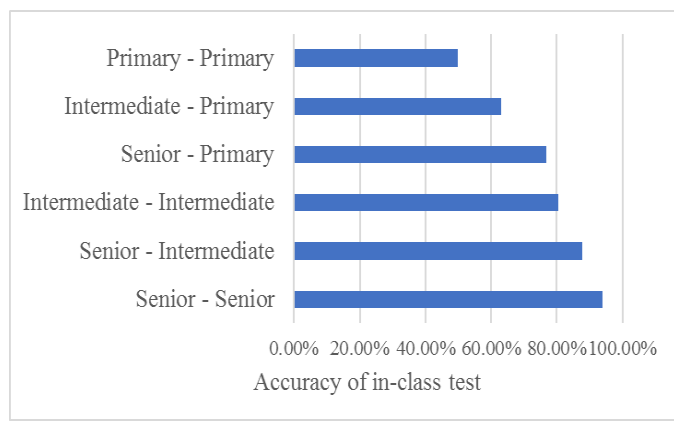

Fig. 4. Results of Different Degree of Combination

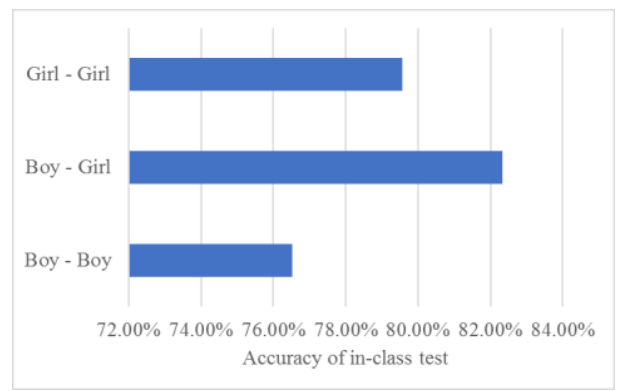

Fig. 3. Results of different gender combinations

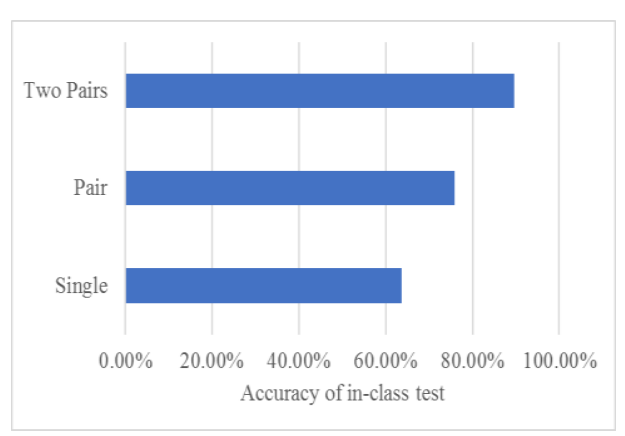

Fig. 5. Test results for different groups

Combination of different ability level has been shown in Fig. 4. In this kind of combination, senior students do have some advantages in the test and the test accuracy is basically in accordance with the upper to the lower. Nevertheless, from the perspective of the experimental process of XPairtise plug-in records, primary and some medium-ability students show high enthusiasm for learning, especially having a great process under the guidance of senior students. Therefore, the Senior - Primary combination works best because the relatively low-ability student can be guided by the relatively senior student

From the effect of the groups divided by number, as is shown in Fig. 5, the form that a pair of two individuals - two pairs of four individuals as a group have the best efficiency, while one person alone is the worst. From the communication records in XPairtise plug-in, we can found that in the process of experiment, the two individuals as a pair have the best efficiency and two pairs as a group are always prone to find out new problems when discussing on XPairtise plug-in. There will be some promotions in the original ability so that the effect is the best.

\section{CONCLUSIONS}

This paper puts forward the concept of pair learning, and through XPairtise plugins, distributed pair programming learning experiment was carried out. On this basis, we can imagine that other content of pair learning can also be applied in distributed application. Office is now supporting collaborative editing documents, editing the document at the same time can be realized through a shared workbook so that work efficiency has been improved. Through functions like this, it can also help people with office online learning. In more computer software, similar functional plugins can be developed as well, which helps people study the usage of this software. In other aspects of learning, through the integration of screen sharing, speech, conversation and antiinterference function of the system, online learning is a kind of excellent method. For example, in some online learning platform, pair learning labs can be constructed to explore the network course, etc.

\section{REFERENCES}

[1] L. Williams: Pair programming illuminated (Addison-Wesley, UK 2003).

[2] Wenhua Jiang: See the world with game thinking (Zhejiang University press, China 2014) .

[3] Kagan and Spencer: Kagan Cooperative Learning (Kagan Publishing, UK 2009).

[4] Xin Wang, Shuqin Bai: China Higher Education Research, 06(2014), p.102-106.

[5] Robert M. Gagn. Reviews: American Educational Research Journal, Vol. 6 (1969) No.2, p.287-290.

[6] P. L. Broadhurst:Acta Psychologica, Vol.16 (1959), p.321-338.

[7] P. Baheti, E. Gehringer, D. Stotts: Extreme Programming and Agile Methods-XP Agile Universe, Vol.2418 (2002), p. 208-220.

[8] Bedrettin Yazan:Creative Dialogue: Talk for Thinking in the Classroom. (European Journal of Teacher Education, London and New York 2011), p.251-254. 